'3Rs alternatives' refers to the replacement, reduction, and refinement of animal use in research, testing, teaching, and exhibition.

The Animal Welfare Act (AWA) and its

regulations require a researcher to consider alternatives to procedures that may cause more than momentary pain or distress to animals and to avoid unnecessarily duplicating previous experiments (see AWA references below). A thorough alternatives literature search can identify information to meet these requirements.

Animal Welfare Act (AWA) and Regulations https://www.nal.usda.gov/animal-health-andwelfare/animal-welfare-act

AWA: United States Code Title 7 - Agriculture Chapter 54 - Transportation, Sale, and Handling of Certain Animals Sec. 2143 (a)(3)(A-B)

AWA Regulations: Code of Federal Regulations Title 9 - Animals and Animal Products, Chapter 1, Subchapter A - Animal Welfare

Sec. $2.31(d)$

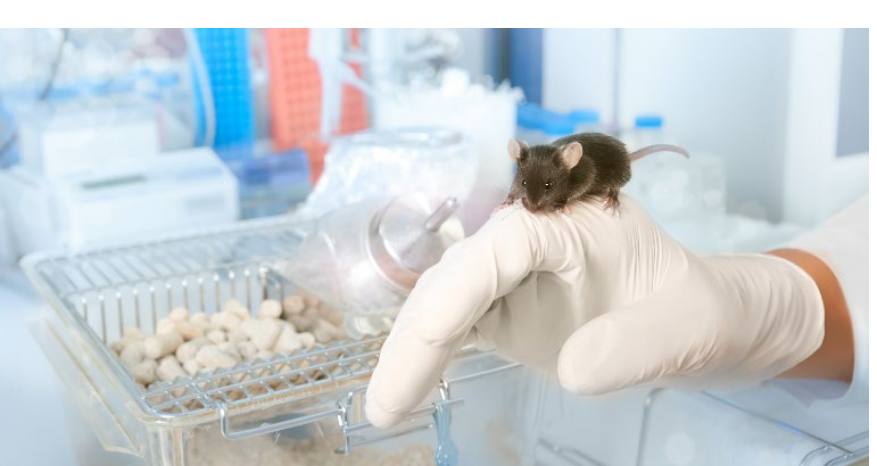

\section{Who We Are}

The Animal Welfare Information Center (AWIC) was established in 1986 as part of the U.S. Department of Agriculture's (USDA) National Agricultural Library (NAL) in Beltsville, Maryland. As a library service, AWIC provides information, products, and services related to the improved care and use of animals in research, teaching, testing, and exhibition as described in the Animal Welfare Act.

AWIC was mandated as part of the 1985 amendment to the Animal Welfare Act [7 U.S.C. § $2143(\mathrm{e})]:$

The Secretary [of Agriculture] shall establish an information service at the National Agricultural Library. Such service shall, in cooperation with the National Library of Medicine, provide information-

- pertinent to employee training;

- which could prevent unintended duplication of animal experimentation as determined by the needs of the research facility; and

- on improved methods of animal experimentation, including methods which could--

o reduce or replace animal use; and

o minimize pain and distress to animals, such as anesthetic and analgesic procedures.

\section{Contact Us}

Phone: (301) 504-6212

Email: awic@usda.gov

Web: https://www.nal.usda.gov/programs/awic

USDA is an equal opportunity provider, employer, and lender. September 2022

\section{USDA}

United States Department of Agriculture

National Agricultural Library,

Animal Welfare Information Center

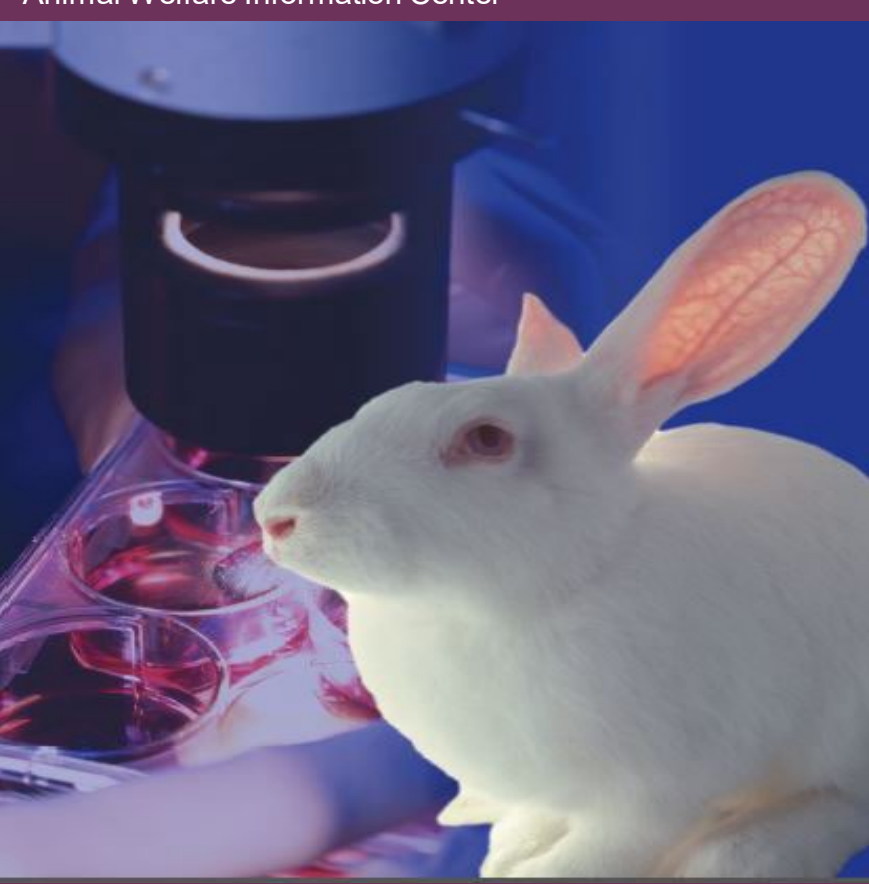

3RS ALTERNATIVES LITERATURE SEARCHING

https://www.nal.usda.gov/services /literature-searching-animal-usealternatives 


\section{Conducting Literature Searches: Considering 3Rs (Replacement, Reduction, Refinement) Alternatives}

1: Identify Areas to Potentially Implement Alternatives

Find areas of the protocol that could potentially incorporate the 3Rs. Think about:

- The protocol's objective and study uniqueness

- Animal used and other animal/non-animal models

- Number of animals required

- Animal housing, care and painful/stressful procedures

\section{2: Finding Databases and Grey Literature} Resources

Search multiple databases! No database covers all journals, subject areas, or types of materials. Talk to your library to find out what databases are available at your institution. Don't forget to search non-database resources as well.

\section{Databases Useful for Finding 3Rs Information**}

- AGRICOLA

- PubMed

- Web of Science

- Scopus

- BIOSIS

- Zoological Record

- EMBASE

\section{Rs Web Resources**}

- AWI Enrichment and Refinement Databases: https://awionline.org/content/enrichment-andrefinement-databases

- JHU CAAT: https://caat.jhsph.edu/

- ICCVAM: https://ntp.niehs.nih.gov/go/regaccept

- NC3Rs: https://www.nc3rs.org.uk/

- NICEATM: https://ntp.niehs.nih.gov/whatwestudy /niceatm/index.html

- NORECOPA: https://norecopa.no/

- NA3RsC: https://www.na3rsc.org/

** Mention of commercial databases or external organizations does not constitute endorsement or imply preference by the U.S. Department of Agriculture.

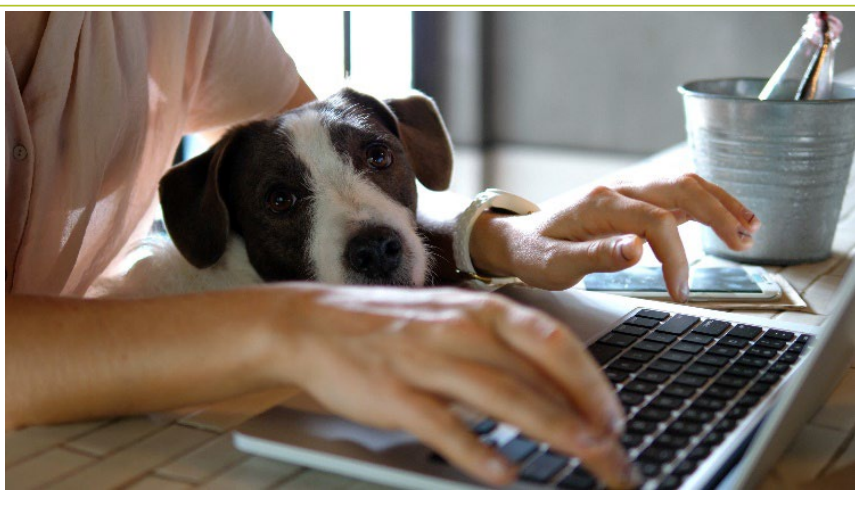

\section{3-4: Developing a Search String}

The search string consists of multiple components:

- Scientific terms related to the research protocol

- Animal terms (if applicable)

- 3Rs alternatives terms relevant to the protocol

- Search logic: Boolean operators, parentheses, quotation marks, truncations, etc. (use AWIC's Database Search Operator Reference Guide for assistance:

https://www.nal.usda.gov/sites/default/files/pag e-files/AWIC-database-cheat-sheet.pdf)

Consider synonyms, acronyms, alternate spellings and variations of the words (such as tenses).

Search String Example in Web of Science:

$\mathrm{TS}=(($ recover* OR assess* OR collect* OR analy* OR process* OR sampl* OR measur*) NEAR/5 (tissue* OR "tissue sample*" OR fluid* OR "fluid sample*" OR "blood sample*” OR "urine sample*”))

\section{Rs Keyword Examples}

- analgesic or analgesia or pain medication

- anesthesia or anaesthesia or anesthetic

- housing or enclosure or caging

- welfare or well-being or wellness

- organoid OR spheroid OR 3D cell culture

- organs-on-chips or microfluidic device or microphysiological system

- repository or data reuse or sharing or repurposing

See our webpage for other examples

\section{5: Modifying a Search String}

Literature searching is a trial-and-error processif you find that your initial search string brings back a lot of irrelevant information, it might be time to modify your search string. Here are some quick ways to modify a search string:

- Change what field you're searching (e.g., title vs. title/abstract)

- Limit by publication year to decrease the number of citations you are receiving

- Remove irrelevant search terms using the Boolean operator 'NOT'

- Narrow the search by replacing general terms for terms specific to the disease, method, procedure, etc., that you're studying

\section{6: Records and Citation Management}

- Save your search strings, databases searched, and years of search in a word document

- Export and save relevant citations into a citation manager (EndNote, Zotero, etc.) to help organize and quickly import citations into research papers

\section{Tips for IACUC Members}

- Ensure protocol forms clearly state what information is required from the researchers

- Inform researchers of available information resources and how to access them

- Encourage researchers to work with librarians and statisticians

- Here are some RED FLAGS to look out for in a literature search:

- Only 1 database searched

- The term "alternatives" is used alone

- Keywords listed are not relevant to protocol

- Keywords and search logic linked incorrectly

(e.g., wrong Boolean operators)

- Search doesn't cover adequate time period 